\title{
Research on Protection and Repair Technology of Historical Building
}

\author{
Qianli Zhang \\ Macao University of Science and Technology, Hubei University, Hubei, Wuhan, 430062
}

Keywords: historic building; protection; repair

\begin{abstract}
The historical and cultural development of China's five thousand years has left us with a large number of architectural heritage and most of the historical buildings have actual needs for the continued use of functions. Therefore, historical buildings are advocated to be repaired on the basis of protection. The main significance of the protection and restoration of historical buildings is to maintain the function of the building and to make people understand the significance of historical tributes to modern civilization. Therefore, to repair historical buildings, first of all, it is necessary to ensure that their architectural style and materials are used as much as possible, and minimize the traces of manual repair. It can be seen that to protect and repair historical buildings, it is necessary to have a deep understanding of their building structure, and then repair them according to relevant photos and records. The paper mainly discusses the protection and repair technology of historical buildings.
\end{abstract}

\section{Introduction}

Historical architecture is an important carrier for human inheritance of human civilization. Modern people should take responsibility for protecting historical buildings from damage and repairing the natural damage of buildings, so that historical culture can be further enriched through the harmonious integration of historical buildings into human modern life.

\section{The Significance of Historical Building Protection and Restoration}

To a certain extent, historical architecture is a witness to urban development, an embodiment of urban cultural and artistic atmosphere and architectural style. Therefore, it is of great significance to protect and repair historical buildings, as follows:

First of all, the protection and restoration of historical buildings is conducive to the inheritance of history and culture. Studying historical buildings can more accurately understand the living standards and cultural connotations of people in the historical era. It can be said that ancient architecture is the epitome of a certain historical period and the carrier of memory history. If historical buildings are destroyed, then the ancient civilization they represent Cultural connotations will also be gradually forgotten, and the development of historical culture will have a fault. Second, enhance national self-confidence and inspire national self-esteem. China's historical buildings have a very high value of art laboratory, the world-famous Beijing Forbidden City, the Yuanmingyuan, which has been damaged but still reminds people not to forget the national humiliation, these are the precious legacy left by the ancestors, and Chinese children will also be proud and proud of these great buildings. Third, historical buildings have promoted the development of the urban tourism industry. The improvement of people's material living standards makes their spiritual pursuit more urgent. The vigorous development of the tourism industry is the best witness to people's spiritual needs. As an important tourism resource of a city, historical architecture is an important measure for the city to self-promote and develop the tourism industry. For tourists, it is an important spiritual sustenance to be able to taste history through historical buildings and dialogue with ancient civilizations. Finally, the artistry and creativity of historical buildings have high reference value. Whether it is a technical achievement or an artistic achievement, China's historical architecture is highly representative. Whether it is layout, materials or architectural techniques and styles, it reflects the rich beauty of the East and the artistic features. These are very good examples for the 
design and construction of modern architecture.

\section{The Protection and Repair of Historical Building Walls}

\subsection{Wall Cleaning.}

The cleaning of historical building walls should fully consider the structure and composition of the wall materials, understand the composition and structure of the dirt, determine the chemical and physical properties of the dirt, and also fully consider the cost of cleaning, environmental factors, and the building. The decorative parts, the length of the construction period and other factors finally determine a scientific, reasonable and low-cost cleaning solution. In the specific cleaning, select the applicable machinery according to different facade walls, then prepare the cleaning agent, and carry out the cleaning operation in the order from top to bottom. When cleaning, it is necessary to conduct a comprehensive survey of historical buildings. For important historical buildings, construction experts should also provide guidance and suggestions to avoid damage to the walls caused by improper cleaning. After the preparation of the cleaning agent is completed, the solution with less influence and less damage is selected for test cleaning, and the cleaning range is further expanded after the confirmation is correct. For a historic building with a variety of building materials, a targeted cleaning method needs to be selected depending on the surface to be cleaned. After the cleaning is completed, it should be exposed to the natural environment for one to two months. If conditions permit, it can be exposed for one year to make a more accurate evaluation of the cleaning effect of the method.

Commonly used cleaning methods for historical building walls include water washing method, grinding method, dressing method, laser method and chemical method. Among them, grinding method, chemical agent method and water washing method are applied in a wider range, but each has its own advantages and disadvantages, such as grinding method. The original wall surface can be recovered completely, but the surface of the component is easily damaged and the cleanliness is low; although the chemical agent method can obtain a good cleaning effect, the cost is high and the environment is polluted. Although water cleaning is minimally destructive and less polluting, it has a narrow application range and is mainly applied to limestone and low-hardness brick masonry structures. The dressing method uses some adsorbable materials to absorb soluble salts inside the stone, such as clay. Or paper, cotton fiber, etc., and then rinse with water, this method can remove the residue of harmful chemicals, but also has the problem of narrow application range, mainly used to remove the dirt inside the stone. The laser method is a new type of cleaning method. It mainly uses high-energy laser beam to illuminate the surface of the wall. Contaminants such as dirt, rust, and coating will be peeled off or evaporated instantaneously under the action of the laser beam, and finally achieve the purpose of rapid cleaning. . However, the application of laser method is relatively expensive, so it is mainly applied to buildings with historical value and high cultural value.

\subsection{Repair of the Wall.}

Different wall structures need to adopt different repair methods. There are many brick and wood structures in China's historical buildings. Due to the space below, the wall repair of brick and wood structures is mainly discussed. The causes of damage to the brick wall structure include natural factors and human factors. Natural factors include weathering, rain erosion, foundation settlement, earthquake disasters, etc. Human factors mainly include smearing, scoring, mortar covering, and improper restoration. Different repair methods are used for different causes of damage. At present, the commonly used wall repair methods include brick powder repair, paint coating jointing, brick repair, external imitation surface, brick wall reinforcement and other methods. For the wall with severe cracks or misalignment, it can be filled and repaired with resin mortar first, then fixed with steel plate and steel bar. Finally, the interface between the steel plate and the wall is filled with epoxy resin. If the wall damage is not serious, it can be repaired by epoxy resin. With the continuous development of historical building restoration technology, the application of some new 
materials is becoming more and more extensive. For example, fiber reinforced composite materials have been widely used in the reinforcement and repair of concrete buildings, which mainly supplement carbon fiber, glass fiber and barbed wire. The strong material is applied between the original brick wall and the stucco layer to achieve the purpose of enhancing the tensile strength of the wall. However, fiber reinforced composites are not suitable for weathering or chalking.

\subsection{Damp-proof Treatment of the Wall.}

The moisture-proof treatment of historical building walls should accurately analyze the causes of moisture generated by the wall, mostly due to climatic conditions and geographical factors, such as high groundwater level, strong precipitation weather, relatively high relative humidity, etc., and some are the building itself. There are defects in the design, such as improper drainage, pipe blockage, water leakage, etc. Therefore, different moisture-proof treatment methods should be selected according to different situations in the actual wall moisture-proof treatment process. At present, the main building repairs mainly use the injection moisture-proof method to prevent the wall from being damp. The main purpose is to drill holes along the brick joints with the punching machine, and then use the injection pressure barrel to inject the waterproof paste into the brick holes, and the waterproofing agent carries the wall under the action of capillary action. In the body, the cracks in the wall penetrate, flow, and diffuse, and the capillary coefficient of the brick body decreases to finally achieve the purpose of preventing moisture from the wall. In addition, we must fully consider the ventilation system and drainage system of historical buildings, and do a good job of maintenance and protection for daily use.

\section{The Protection and Repair of Historical Building Components}

\subsection{Partial Component Replacement.}

For historical buildings with low protection requirements and severe structural damage, structural replacement parts can be used to improve the performance of the overall structure. However, the partial replacement component should be as close as possible to the concealed structural part, so as not to affect the overall appearance of the structure. For example, the daughter wall of Wangfujing Dongtang in Beijing, the original structure is the continuous arch vouchers of bricks and discs. Due to the thin structure of the wall, the wall collapses when the parapet blockage is removed. In this case, the repair is carried out. When the transparent hole is restored, the reinforced concrete slab is buried in the upper part of the continuous arch vouch, and the reinforced concrete main column is buried in the brick slab. The column and the reinforced concrete slab form the beam-column system, and the carrying capacity of the parapet wall is greatly improved. Of course, for some historical buildings with high protection requirements, it is necessary to avoid replacing structural members.

\subsection{Partial Restoration and Reinforcement of Components.}

Some historical buildings will be seriously damaged or missing due to factors such as social development and history. For this situation, the historical image files can be used to reconstruct the components locally to obtain better architectural appearance. If the damage is not serious, you can restore it according to the picture. Some historical buildings have problems of insufficient carrying capacity, mostly due to factors such as unreasonable design and the influence of natural factors. Therefore, some parts of the building should be reinforced. The reinforcement treatment mainly aims to improve the bearing capacity and seismic performance of the building by improving the performance of the beam, column and other bearing capacity of the building structure. Common reinforcement methods include increasing the section method, prestressing method, reinforcing steel support reinforcement method, and pasting.

\subsection{Repair of Wooden Components.}

There are a large number of wooden components in the historic buildings of our country, and the wooden components are more easily damaged than the clear water brick structure, so the repair of 
the wooden components should be strengthened. The repair of wooden components should be carried out in accordance with the principle of minimizing the number of disassembly and replacement, and retaining the original appearance of the building to the utmost extent. In the protection and repair of wooden components, chemical reinforcement is a widely used one. It mainly uses related materials to reinforce or bond wood components to achieve the purpose of repair. This method is often used for damage to wooden components. Epoxy resin is a commonly used material for repairing wood components. It has the advantages of high strength, good bonding performance and low shrinkage. It has excellent water resistance and chemical resistance and can be used for surface caulking of wooden structures. , hole filling, etc. For example, cracks in wood components are slightly filled with epoxy resin and then reinforced with stainless steel iron hoops. If the crack is wide, the decay of the wood components should be removed first, then the surface of the structure should be sanded with sandpaper, and finally made of the same material. Structures of the same shape are tightly packed with epoxy tree fingers and modified to maintain a flattened effect with the original structure. In addition, for some small-scale historical buildings, the wooden pillar structure is often used. Due to factors such as unreasonable material selection and acquired biological damage, the phenomenon of core defects may occur. For this case, chemical material casting should be adopted. Reinforcement method for repair, such as the thickness of the surface of the wood column structure is more than $50 \mathrm{MM}$, and it can be reinforced with unsaturated polyester resin.

\section{Conclusion}

In short, with the continuous development of social material civilization, people's pursuit of spiritual civilization is getting higher and higher. Historical architecture is an important part of historical and cultural heritage. It is not only the inheritance of history and culture, but also an important tourism resource, and it is also the spirit of people. The sustenance of life and the promotion of historical and cultural carriers are of great significance for the protection and restoration of historical buildings. In the actual work, we must protect and repair the wall and structure, try to apply some new technologies and new materials, give full play to the role of modern science and technology in the protection and restoration of historical buildings, and effectively improve the quality of repair.

\section{Acknowledgement}

This article is the research result of the foundation item, which belongs to Hubei Intangible Cultural Heritage Protection Center in 2017(Hubei institute of fine art).The number of the project is FY-2017-03.

\section{References}

[1] Cao Jianwei, Jiang Xiangqi. Research Status and Prospect of FRP Reinforcement and Repair of Wood Structure [J].Shanxi Architecture, 2006(2):54

[2] Chen Jin, Yan Jianwei. Looking for the Lost Building: Talking from the Protection of Nordic Architectural Heritage [J]. World Architecture, 2012(1): 77

[3] Chen Zhihua. Introducing several international documents on cultural relics and historical urban protection (I) [J]. World Architecture, 1989(2): 85-86

[4] Luo Zhewen. The Principles of Ancient Building Maintenance and the Application of New Materials and New Technologies_-Also on the Chinese Characteristics of Cultural Relics Construction Protection and Maintenance [J]. Cultural Relics Protection Engineering, 2006(04): 152-153

[5] Gao Nianhua. On the restoration of ancient Chinese architecture - an understanding of the 
relevant provisions of the Venice Charter [N]. Chinese Cultural Relics, 2002-7-26 (5).

[6] Yan Junhong. Historical Building Protection Guidelines and Protection Technology Research-The Theory and Practice of Modern Building Protection and Utilization in Shenyang [D]. Tianjin: School of Architecture, Tianjin University, 2007: 46.

[7] Wang Xiang. Application of carbon fiber cloth reinforcement technology in protective historical buildings [J]. Building construction, 2008: 387 